\title{
Nobel Lecture: A half-century with solar neutrinos*
}

\author{
Raymond Davis, Jr. \\ Department of Physics and Astronomy, University of Pennsylvania, \\ Philadelphia, Pennsylvania 19104, USA \\ and Chemistry Department, Brookhaven National Laboratory, Upton, New York 11973, \\ USA
}

(Published 8 August 2003)

Neutrinos are neutral, nearly massless particles that move at nearly the speed of light and easily pass through matter. Wolfgang Pauli (1945 Nobel Laureate in Physics) postulated the existence of the neutrino in 1930 as a way of carrying away missing energy, momentum, and spin in beta decay. In 1933, Enrico Fermi (1938 Nobel Laureate in Physics) named the neutrino ("little neutral one" in Italian) and incorporated it into his theory of beta decay.

The Sun derives its energy from fusion reactions in which hydrogen is transformed into helium. Every time four protons are turned into a helium nucleus, two neutrinos are produced. These neutrinos take only two seconds to reach the surface of the Sun and another eight minutes or so to reach the Earth. Thus, neutrinos tell us what happened in the center of the Sun eight minutes ago. The Sun produces a lot of neutrinos, $1.8 \times 10^{39}$ per second: even at the Earth, 150 million kilometers from the Sun, about 100 billion pass through an average fingernail $\left(1 \mathrm{~cm}^{2}\right)$ every second. They pass through the Earth as if it weren't there, and the atoms in the human body capture a neutrino about every seventy years, or once in a lifetime. As we will see, neutrinos captured me early in my career.

I received my Ph.D. from Yale in 1942 in physical chemistry (Davis, 1942) and went directly into the Army as a reserve officer. After the war, I decided to search for a position in research with the view of applying chemistry to studies in nuclear physics. After two years with the Monsanto Chemical Company in applied radiochemistry of interest to the Atomic Energy Commission, I was very fortunate in being able to join the newly created Brookhaven National Laboratory. Brookhaven was created to find peaceful uses for the atom in all fields of basic science: chemistry, physics, biology, medicine, and engineering.

When I joined the Chemistry Department at Brookhaven, I asked the chairman, Richard Dodson, what he wanted me to do. To my surprise and delight, he told me to go to the library and find something interesting to work on. I found a stimulating review on neutrinos (Crane, 1948). This quote from Crane shows that

\footnotetext{
*The 2002 Nobel Prize in Physics was shared by Raymond Davis, Jr., Riccardo Giacconi, and Masatoshi Koshiba. This is the text of the lecture delivered on behalf of Dr. Raymond Davis, Jr. by his son Andrew Davis on the occasion of the award.
}

neutrino physics was a field that was wide open to exploration: "Not everyone would be willing to say that he believes in the existence of the neutrino, but it is safe to say that there is hardly one of us who is not served by the neutrino hypothesis as an aid in thinking about the beta-decay hypothesis." Neutrinos also turned out to be suitable for applying my background in physical chemistry. So, how lucky I was to land at Brookhaven, where I was encouraged to do exactly what I wanted and get paid for it! Crane had quite an extensive discussion on the use of recoil experiments to study neutrinos. I immediately became interested in such experiments (Fig. 1). I spent the first year working on the recoil of ${ }^{107} \mathrm{Ag}$ from the electron-capture decay of ${ }^{107} \mathrm{Cd}$, but these experiments were inconclusive.

My first successful experiment was a study of the recoil energy of a ${ }^{7} \mathrm{Li}$ nucleus resulting from the electroncapture decay of ${ }^{7} \mathrm{Be}$. In ${ }^{7} \mathrm{Be}$ decay, a single monoenergetic neutrino is emitted with an energy of $0.862 \mathrm{MeV}$, and the resulting ${ }^{7} \mathrm{Li}$ nucleus should recoil with a characteristic energy of $57 \mathrm{eV}$. A measurement of this process provides evidence for the existence of the neutrino. In my experiment, the energy spectrum of a recoiling ${ }^{7} \mathrm{Li}$ ion from a surface deposit of ${ }^{7} \mathrm{Be}$ was measured and found to agree with that expected from the emission of a single neutrino (Davis, 1952). This was a very nice result, but I was scooped by a group from the University of Illinois (Smith and Allen, 1951).

In 1951, I began working on a radiochemical experi-

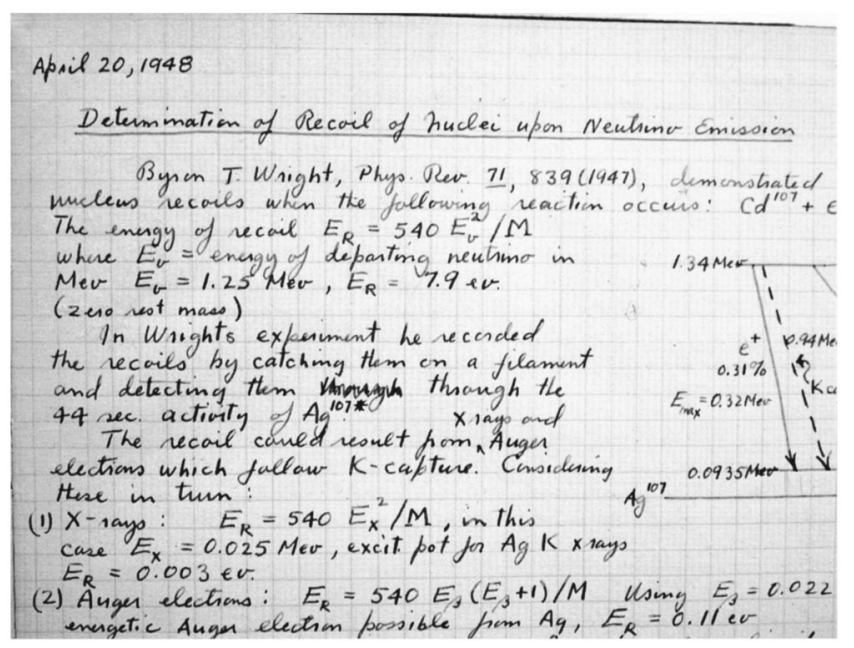

FIG. 1. The first page in my first laboratory notebook at Brookhaven National Laboratory. I was hooked on neutrinos from the beginning. 
TABLE I. Neutrino-producing reactions in the Sun.

\begin{tabular}{|c|c|c|c|c|}
\hline & Reaction & Frequency & Energy (MeV) & Name \\
\hline \multicolumn{5}{|l|}{ PPI } \\
\hline & \multirow{4}{*}{$\begin{array}{c}p+p \rightarrow{ }^{2} \mathrm{H}+e^{+}+\nu_{e} \\
p+e-+p \rightarrow{ }^{2} \mathrm{H}+\nu_{c} \\
{ }^{2} \mathrm{H}+p \rightarrow{ }^{3} \mathrm{He}+\gamma \\
{ }^{3} \mathrm{He}+{ }^{3} \mathrm{He} \rightarrow{ }^{4} \mathrm{He}+2 p\end{array}$} & $99.75 \%$ & \multirow{4}{*}{$\begin{array}{l}0.0-0.42 \\
1.44\end{array}$} & \multirow{4}{*}{$\begin{array}{l}\text { pp } \\
\text { pep }\end{array}$} \\
\hline & & $0.25 \%$ & & \\
\hline & & $100 \%$ & & \\
\hline & & $85 \%$ & & \\
\hline \multicolumn{5}{|l|}{ PPII } \\
\hline & \multirow{3}{*}{$\begin{array}{c}{ }^{3} \mathrm{He}+{ }^{4} \mathrm{He} \rightarrow{ }^{7} \mathrm{Be}+\gamma \\
e^{-}+{ }^{7} \mathrm{Be} \rightarrow{ }^{7} \mathrm{Li}+\nu_{e} \\
p+{ }^{7} \mathrm{Li} \rightarrow{ }^{4} \mathrm{He}+{ }^{4} \mathrm{He}\end{array}$} & $15 \%$ & \multirow{3}{*}{$0.86,0.38$} & \multirow{3}{*}{${ }^{7} \mathrm{Be}$} \\
\hline & & $99.99 \%$ & & \\
\hline & & $100 \%$ & & \\
\hline \multicolumn{5}{|l|}{ PPIII } \\
\hline & $p+{ }^{7} \mathrm{Be} \rightarrow{ }^{8} \mathrm{~B}+\gamma$ & $0.01 \%$ & & \\
\hline & ${ }^{8} \mathrm{~B} \rightarrow{ }^{4} \mathrm{He}+{ }^{4} \mathrm{He}+e^{+}+\nu_{c}$ & $100 \%$ & $0-14.1$ & ${ }^{8} \mathrm{~B}$ \\
\hline
\end{tabular}

ment for detecting neutrinos using a method that was suggested by Pontecorvo (1946): capturing neutrinos with the reaction: ${ }^{37} \mathrm{Cl}+\nu_{e} \rightarrow{ }^{37} \mathrm{Ar}+e^{-}$. Bruno Pontecorvo's short paper was quite detailed, and the method he described, removing argon by boiling carbon tetrachloride and counting ${ }^{37} \mathrm{Ar}$ in a gas-filled counter, has many similarities to techniques I eventually used. Pontecorvo's report (1946) was from the Chalk River Laboratory in Canada and was classified by the U.S. Atomic Energy Commission, because it was feared that the method could be used to measure the power output of reactors. Luis Alvarez (1968 Nobel Laureate in Physics) proposed to use the chlorine-argon reaction to detect solar neutrinos with a large tank of concentrated sodium chloride solution (Alvarez, 1949), but did not choose to pursue the experiment. Since no one else appeared interested in attempting the chlorine-argon neutrino detection method, it seemed a natural and timely experiment for me to work on.

In the Pontecorvo method, neutrino capture on ${ }^{37} \mathrm{Cl}$ makes ${ }^{37} \mathrm{Ar}$, a radioactive isotope that decays back to ${ }^{37} \mathrm{Cl}$ by the inverse of the capture process with a half-life of 35 days. The threshold for the capture reaction is $0.814 \mathrm{MeV}$, meaning that neutrinos with energies of less than $0.814 \mathrm{MeV}$ will not be captured. There are two potential sources of neutrinos: fission reactors and the Sun, both of which were suggested as possible sources by Pontecorvo (1946).

In my first experiment using the ${ }^{37} \mathrm{Cl}^{-37} \mathrm{Ar}$ reaction, I tried to detect neutrinos from a fission reactor, using carbon tetrachloride $\left(\mathrm{CCl}_{4}\right)$ as the target material (Davis, 1955). I exposed a 3800-liter tank of carbon tetrachloride at the Brookhaven Graphite Research Reactor for a month or two, removed the argon and counted it in a small Geiger counter. That reactor did not have a high enough neutrino flux to detect with this target size, so neutrinos were not observed. Furthermore, a reactor emits antineutrinos, and the ${ }^{37} \mathrm{Cl}^{-37} \mathrm{Ar}$ reaction requires neutrinos. It was not clear at that time, however, whether neutrinos and antineutrinos were different particles, nor was it clear how they could differ. After all, there are other examples in nature where the particle is its own antiparticle, such as the photon and the pi-zero.
The background due to cosmic rays was significant, so as part of the experiment, I buried a 3800-liter tank of carbon tetrachloride 5.8 meters underground. From the background-corrected ${ }^{37} \mathrm{Ar}$ count rate, I was able to obtain an upper limit on the solar neutrino flux of 40,000 SNU, a factor of 15,000 above my eventual result of 2.56 SNU (the solar neutrino unit, or SNU, is defined as $10^{-36}$ captures per target atom per second). One reviewer of my paper was not very impressed with this upper limit and commented: "Any experiment such as this, which does not have the requisite sensitivity, really has no bearing on the question of the existence of neutrinos. To illustrate my point, one would not write a scientific paper describing an experiment in which an experimenter stood on a mountain and reached for the moon, and concluded that the moon was more than eight feet from the top of the mountain." It was clear that the Brookhaven reactor was not a powerful enough neutrino source, so in 1954, I built an experiment using 3800 liters of $\mathrm{CCl}_{4}$ in the basement of one of the Savannah River reactors, the most intense antineutrino source in the world.

One can calculate the total capture rate from all fission product antineutrinos by ${ }^{37} \mathrm{Cl}$, presuming neutrinos and antineutrinos are equivalent particles. The sensitivity for detecting neutrinos and the flux at Savannah River were sufficiently high to provide a critical test for the neutrino-antineutrino identity. I did not detect any reactor neutrinos and found that the neutrino capture rate was a factor of five below the antineutrino capture rate. I later did a 11,400-liter experiment at Savannah River that lowered the upper limit for neutrino capture to a factor of 20 below the antineutrino capture rate (Davis, 1958). While I was at Savannah River, Frederick Reines (Nobel Prize in Physics, 1995) and Clyde Cowan and their associates were performing a beautiful experiment, the first detection of a free antineutrino (Cowan et al., 1956; Reines et al., 1960). This experiment was a clear demonstration that the neutrino postulated by Pauli was indeed a real particle. My experiment showed that the neutrino was not its own antiparticle.

As I mentioned earlier, the Sun's energy comes from fusion of four hydrogen atoms to make a helium atom. I 


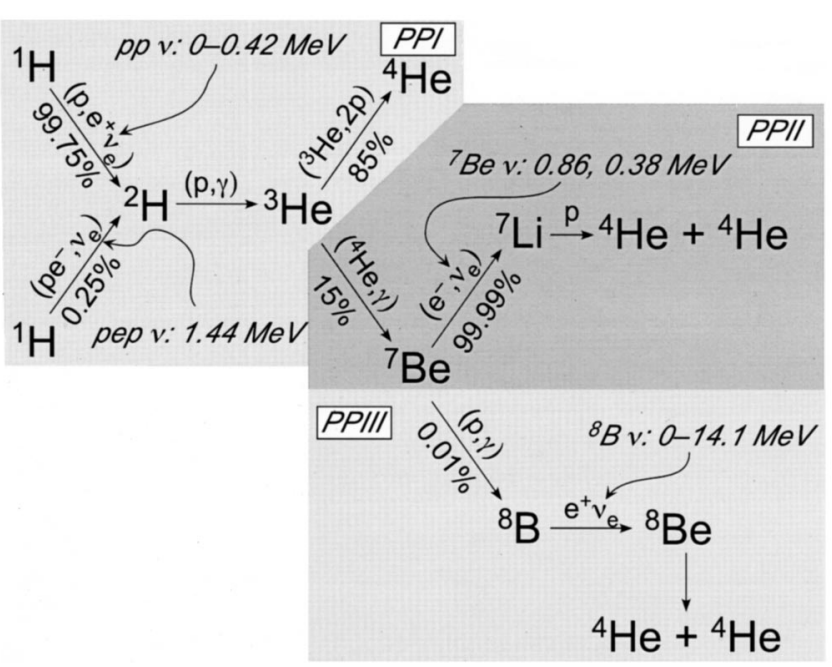

FIG. 2. The main energy-producing reactions in the Sun, along with their associated neutrinos.

would now like to explore the reactions in a little more detail and note which ones can produce neutrinos detectable with the chlorine-argon experiment. The relative rates of nuclear reactions in the Sun are based on detailed modeling of temperatures, pressures, and elemental abundances in the Sun, into which are put laboratory-measured and theoretically calculated rates of appropriate nuclear reactions. What I show in Table I and Fig. 2 represent current best estimates. These estimates have changed with time, but the predicted solar neutrino flux has not changed much since the late 1960s.

Most of the time, two protons react to make a deuteron, a positron, and an electron neutrino, but the neutrinos are below the threshold of the chlorine capture reaction (these neutrinos are known as pp neutrinos). One time out of 400, two protons react with an electron to make a deuteron and a neutrino. These so-called "pep" neutrinos are quite energetic, but there are not very many of them. All the deuterons react with a proton to make ${ }^{3} \mathrm{He}$, and most of the ${ }^{3} \mathrm{He}$ nuclei react with one another to make ${ }^{4} \mathrm{He}$ and two protons. The latter reaction occurs $85 \%$ of the time. This major branch is usually referred to as the PPI chain (Fig. 2, Table I).

Fifteen percent of the time, however, ${ }^{3} \mathrm{He}$ and ${ }^{4} \mathrm{He}$ nuclei react to make ${ }^{7} \mathrm{Be}$. Nearly all of the ${ }^{7} \mathrm{Be}$ then captures an electron to make ${ }^{7} \mathrm{Li}$ and a neutrino. The ${ }^{7} \mathrm{Li}$ captures a proton to make two ${ }^{4} \mathrm{He}$ nuclei, completing the PPII chain, but one in $10,000{ }^{7} \mathrm{Be}$ nuclei captures a proton to make ${ }^{8} \mathrm{~B}$ (Fig. 2, Table I). ${ }^{8} \mathrm{~B}$ decays to ${ }^{8} \mathrm{Be}$, which splits to make two ${ }^{4} \mathrm{He}$ nuclei, a positron, and an electron neutrino, completing the PPIII chain. It is these ${ }^{8} \mathrm{~B}$ neutrinos that produce most of the solar neutrino signal I detected, but there is also some contribution from pep and ${ }^{7} \mathrm{Be}$ neutrinos.

In the 1950s, it was thought that the PPI chain was the only significant source of neutrinos, but the energy of the dominant pp neutrinos was too low to detect with the ${ }^{37} \mathrm{Cl}$ reaction. The branch to make ${ }^{7} \mathrm{Be}$ was thought not to be significant and to occur only $0.015 \%$ of the time. The $\mathrm{CNO}$ cycle, in which four protons make a ${ }^{4} \mathrm{He}$ nucleus, could produce energetic neutrinos from the ${ }^{13} \mathrm{~N}\left(e^{+}, \nu_{e}\right){ }^{13} \mathrm{C}$ and ${ }^{15} \mathrm{O}\left(e^{+}, \nu_{e}\right){ }^{15} \mathrm{~N}$ reactions, but it was not clear that the CNO cycle operated in the Sun. Thus, an experiment to detect solar neutrinos did not seem feasible at the time.

This situation changed dramatically when Holmgren and Johnston (1958) reported that the rate of reaction ${ }^{3} \mathrm{He}+{ }^{4} \mathrm{He} \rightarrow{ }^{7} \mathrm{Be}+\gamma$ is 1000 times greater than previously thought. It was immediately recognized that the path to ${ }^{7} \mathrm{Be}$, now about $15 \%$ of the time rather than one in 7000 , was significant. Furthermore, the ${ }^{7} \mathrm{Be}$ could react with a proton and become ${ }^{8} \mathrm{~B}$. These two radioactive products, ${ }^{7} \mathrm{Be}$ and ${ }^{8} \mathrm{~B}$, are sources of energetic neutrinos. The relative rates of electron and proton capture on ${ }^{7} \mathrm{Be}$ were not measured, but estimated rates allowed the possibility that virtually all ${ }^{7} \mathrm{Be}$ captured a proton to make ${ }^{8} \mathrm{~B}$. The decay of ${ }^{8} \mathrm{~B}$ to ${ }^{8} \mathrm{Be}$ would be accompanied by neutrinos with typical energies of $7 \mathrm{MeV}$. W. A. Fowler and A. G. W. Cameron immediately relayed to me these developments. They pointed out that the neutrino flux from these sources could perhaps be easily observed by the chlorine-argon method (Cameron, 1958; Fowler, 1958). The initial estimate was very optimistic, several captures per day if all the ${ }^{7} \mathrm{Be}$ captured a proton to make ${ }^{8} \mathrm{~B}$, so we set up a 3800 -liter tank of perchloroethylene $\left(\mathrm{C}_{2} \mathrm{Cl}_{4}\right)$ in the Barberton Limestone Mine near Akron, Ohio. Our initial experimental results were disappointing: we didn't detect any clear signal of neutrinoproduced ${ }^{37} \mathrm{Ar}$.

Bahcall (1962) calculated the ${ }^{7} \mathrm{Be}$ electron capture rate after I pointed out the importance of knowing this reaction rate. At about this time, Kavanagh (1960) measured the ${ }^{7} \mathrm{Be}(p, \gamma)$ reaction rate, which is the one that produces ${ }^{8} \mathrm{~B}$, and found it to be disappointingly low. It is the ratio of the two reactions that consume ${ }^{7} \mathrm{Be}$ that was so discouraging. With most of the ${ }^{7} \mathrm{Be}$ capturing an electron to make ${ }^{7} \mathrm{Li}$ rather than capturing a proton to make ${ }^{8}$ B (Fig. 2), it would be very hard for us to detect neutrinos. Reines (1960) wrote "The probability of a negative result even with detectors of thousands or possibly hundreds of thousands of gallons of perchloroethylene tends to dissuade experimentalists from making the attempt."

The situation brightened considerably in 1963, when John Bahcall carefully calculated the capture rate of ${ }^{8} \mathrm{~B}$ neutrinos and showed that the rate was 20 times higher than previously expected. This led us to propose the large chlorine experiment. The theory and experimental approach were laid out in back-to-back papers (Bahcall, 1964; Davis, 1964). The theory provided guidance as to how large a tank to build: the predicted production rate was 4 to $11{ }^{37} \mathrm{Ar}$ atoms per day in 100,000 gallons (378,000 liters) of perchloroethylene. Our measurement of the cosmic-ray muon background at Barberton told us how deeply it needed to be buried.

The events of the late 1950s and early 1960s ultimately led Brookhaven National Laboratory, with support from the chemistry office of the Atomic Energy Commission, to build a 100,000-gallon chlorine-argon neutrino detector in the Homestake Gold Mine, in Lead, South Da- 


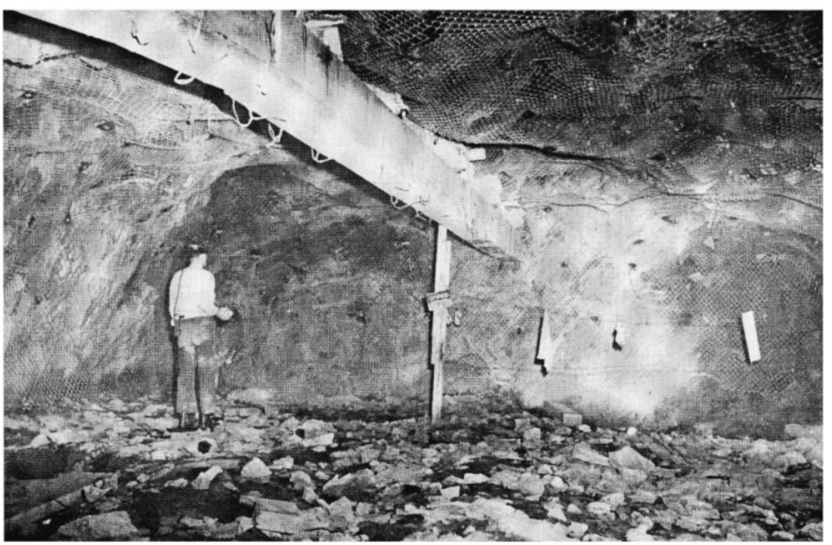

FIG. 3. The large room where the tank will be installed in the Homestake Mine is shown about half-excavated, in the summer of 1965. From Sharp Bits, September 1965, published by the Homestake Mining Company.

kota. The construction of the experiment in 1965-1966 was detailed in a series of articles in Sharp Bits, published by the Homestake Mining Company for its employees. A large chamber and a smaller control room were excavated at the 4850 -foot $(1478 \mathrm{~m})$ level of the mine. In Fig. 3, the excavation of the main chamber is shown half finished. The tank was built by the Chicago Bridge and Iron Company, which was known for building municipal water tanks, reactor containment vessels, space simulation chambers, and other kinds of tanks. The tank had to be built in pieces small enough to fit in the mine hoist. Figure 4 shows one end of the tank laid out on the floor of the big tank room of the mine like flower petals. All welds were checked by use of x-rays. In Fig. 5 an $x$-ray generator is being placed at the axis of

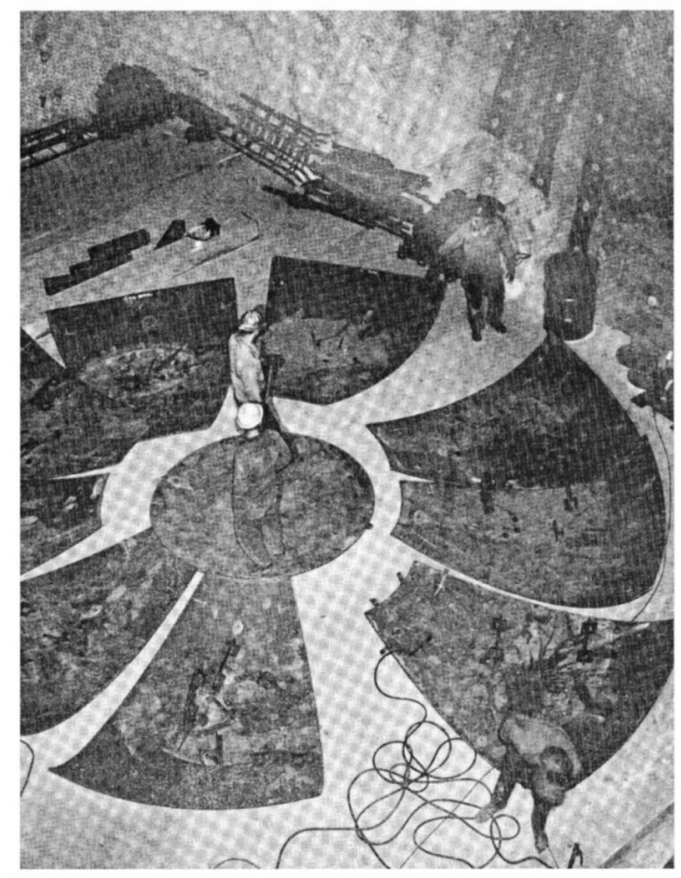

FIG. 4. The pieces for one end of the tank are laid out on the floor of the big room. From Sharp Bits, June 1966.

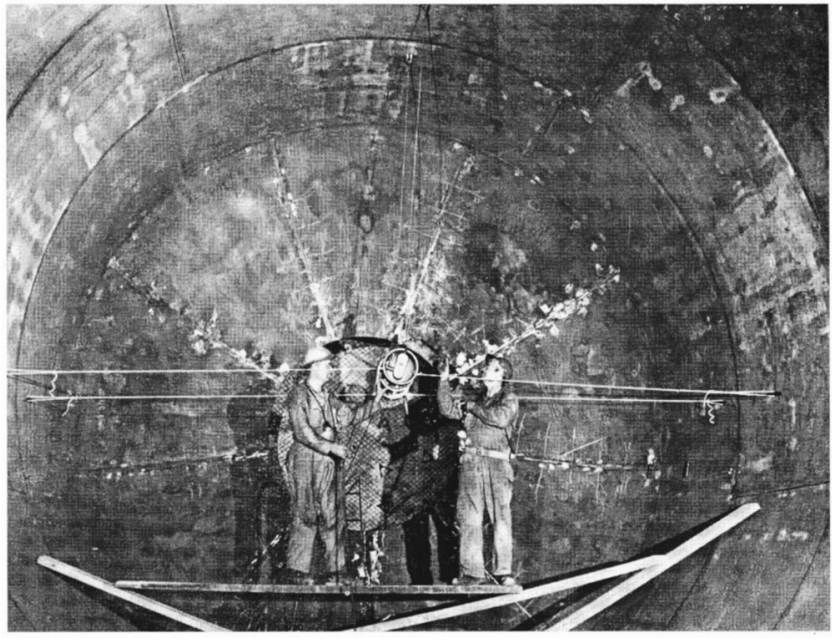

FIG. 5. An x-ray source was placed at the axis of the tank and $\mathrm{x}$-ray film was attached to the outside of the tank, in order to test all of the welds. From Sharp Bits, June 1966.

the tank. X-ray film was attached to the tank all the way around a weld and then exposed to x-rays from the source inside the tank. The next step was to vacuum leak-test the tank. The entire tank was pumped out with a 12-inch diffusion pump (Fig. 6) and then leak-tested using a helium leak detector. In order to extract argon from the tank, helium is bubbled through the perchloroethylene. This was done very efficiently using a device called an eductor, shown in Fig. 7 being tested in the Brookhaven Lab swimming pool. The swimming pool is filled with water, not perchloroethylene. Figure 8 shows the eductors inside the completed tank.

Figure 9 shows the layout of the experiment. During an argon extraction, which occurred every two to three months, two large pumps were used to circulate perchlo-

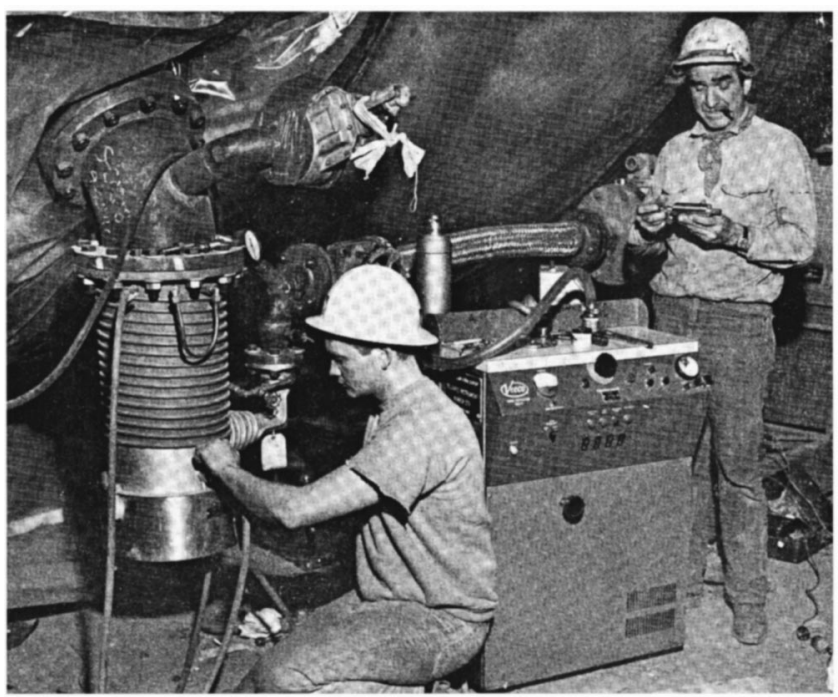

FIG. 6. The entire tank was vacuum leak-tested by Chicago Bridge and Iron engineers. A small mass spectrometer capable of sensitive detection was connected to the vacuum pump and helium was introduced around the tank and all the plumbing. From Sharp Bits, September 1966. 


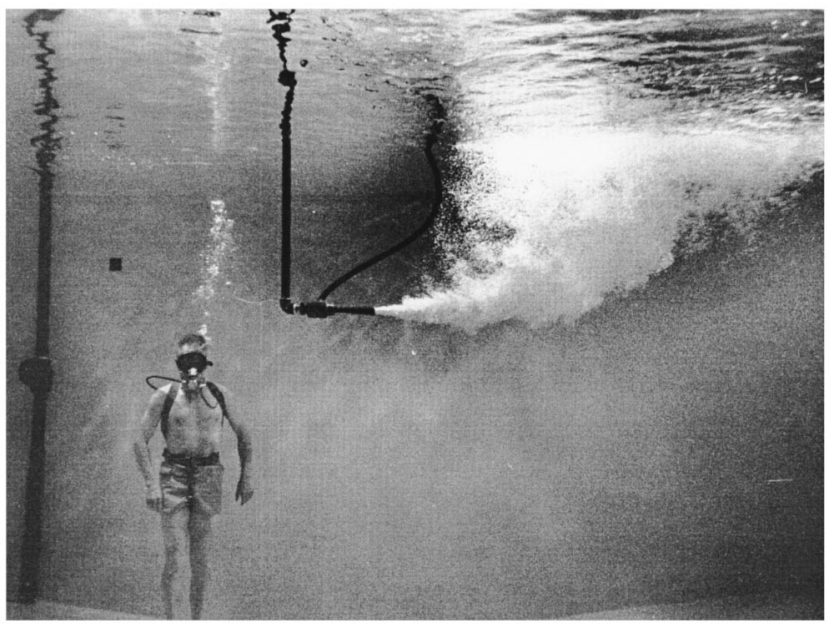

FIG. 7. The eductors, which allowed efficient mixing of helium and perchloroethylene, are being tested here in the swimming pool at Brookhaven, using air and water. From the Brookhaven National Laboratory Website http://www.bnl.gov/ bnlweb/raydavis/images/hires/11-755-64.jpg

roethylene through the eductors. The perchloroethylene flow rate was about 1500 liters per minute. While helium was bubbled through the perchloroethylene, the helium atmosphere in the top 5\% of the tank was circulated at 17,000 liters per minute through the control room, for extraction of argon. A large condenser near the big tank froze out perchloroethylene and a charcoal trap in the control room (Fig. 10) trapped argon. All of the plumbing was controlled from the control room (Fig. 11) About $95 \%$ of the argon in the tank was removed in 20 hours of circulation. After the argon was trapped, it was purified and placed in tiny proportional counters that have internal volumes of 0.25 or $0.5 \mathrm{~cm}^{3}$ (Fig. 12). In the

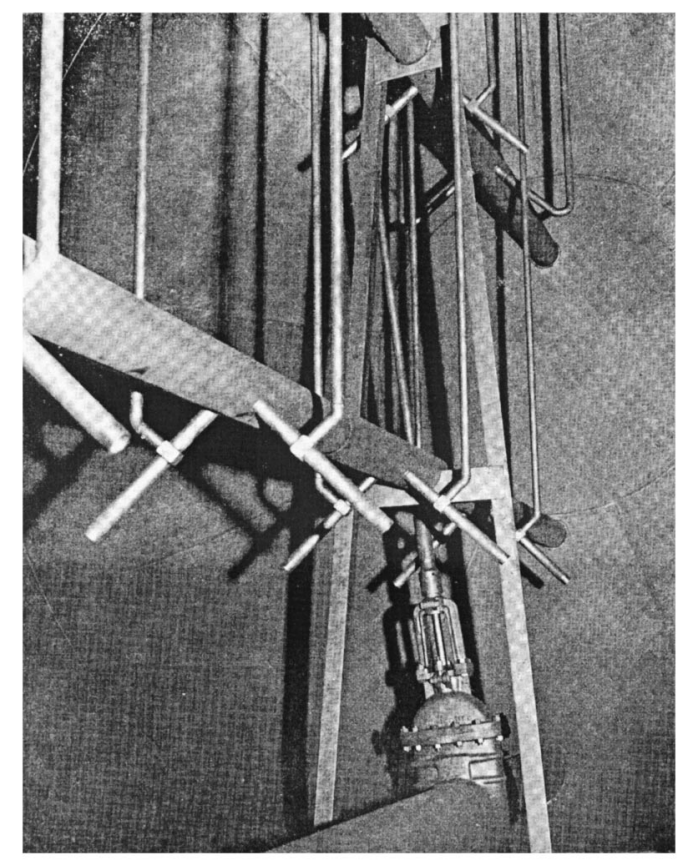

FIG. 8. A double row of eductors were installed in the tank. From Sharp Bits, September 1966. early years, the samples were counted at Brookhaven, inside shields built from battleship gun barrels made before the atomic bomb (Fig. 13). Later, the counting apparatus was moved to the Homestake Mine to take advantage of the much better shielding from cosmic rays deep underground.

In order to forecast as accurately as possible the rate of solar neutrino capture in the Homestake detector, it was necessary to measure the production cross sections of the neutrino-producing reactions, and derive their rates in the interior of the Sun. This great effort was largely carried out at the California Institute of Technology under the leadership of William A. Fowler (Nobel Prize in Physics, 1983). Many nuclear astrophysicists and astronomers contributed to the basic physics that supported this early effort in solar neutrino astronomy. Our task at Brookhaven was far simpler and we (Don Harmer, Ken Hoffman, and myself) had the fun of building a large detector and making it work.

The Homestake experiment became operational in 1967. Even after the first run, it was clear the solar neutrino flux was lower than predicted. On August 11, 1967, I wrote to Willy Fowler, who was on a sabbatical at Cambridge University.

\section{Dear Willy,}

I do have a preliminary result from our first good run. The sample was taken Jun 22nd and counting has continued until today. I am now removing the sample and will rerun background. So we do have a result and during the last few weeks I have told a few people that are interested. I have, of course, had many telephone conversations with John.

The tank and helium purge system seems to work quite well. The first job was to clear out the air argon and reduce its level to about $1 \mathrm{cc}$ before we could make a sensitive measurement. This was accomplished finally and the first good measurement started May 5th. At this time $0.115 \mathrm{~cm}^{3}$ of $\mathrm{Ar}^{36}$ carrier was introduced and the first irradiation started. It ran 48 days, and on June 22nd we recovered the $\mathrm{Ar}^{36}$ with a 94 percent efficiency. The sample was counted yielding the pulse height spectrum on the enclosed sheet. As you see, there is no visible peak at $\mathrm{Ar}^{37}(3.0 \mathrm{keV})$. The background for this counter run just before the sample is shown also on the enclosed sheet. Comparing these we can obtain the following results:

Argon from $10^{5}$ gal tank $=16 \pm 4$ counts (tot. $39.7 \mathrm{~d}$ ) Background $=4 \times(39.7 / 11.5)=14 \pm 4$ counts $($ for 39.7

d)

Increase $=2 \pm 5$ counts

Using: $2.1 \times 10^{30} \mathrm{Cl}^{37}$ atoms in tank

Counter efficiency $\approx 0.50$

Then, $\Sigma \phi \sigma=(0.2 \pm 0.4) \times 10^{-35} \mathrm{sec}^{-1}$ $\leqslant 0.6 \times 10^{-35} \mathrm{sec}^{-1}$

Using $\sigma\left(\mathrm{B}^{8}\right)=1.35 \times 10^{-42}$ (Bahcall)

$\phi_{\mathrm{B}^{8}} \leqslant 0.5 \times 10^{7} \mathrm{~cm}^{-2} \mathrm{sec}^{-1}$

This limit is quite low, but according to the latest opus from Bahcall and Shaviv the $\mathrm{B}^{8}$ flux is 1.4(1 


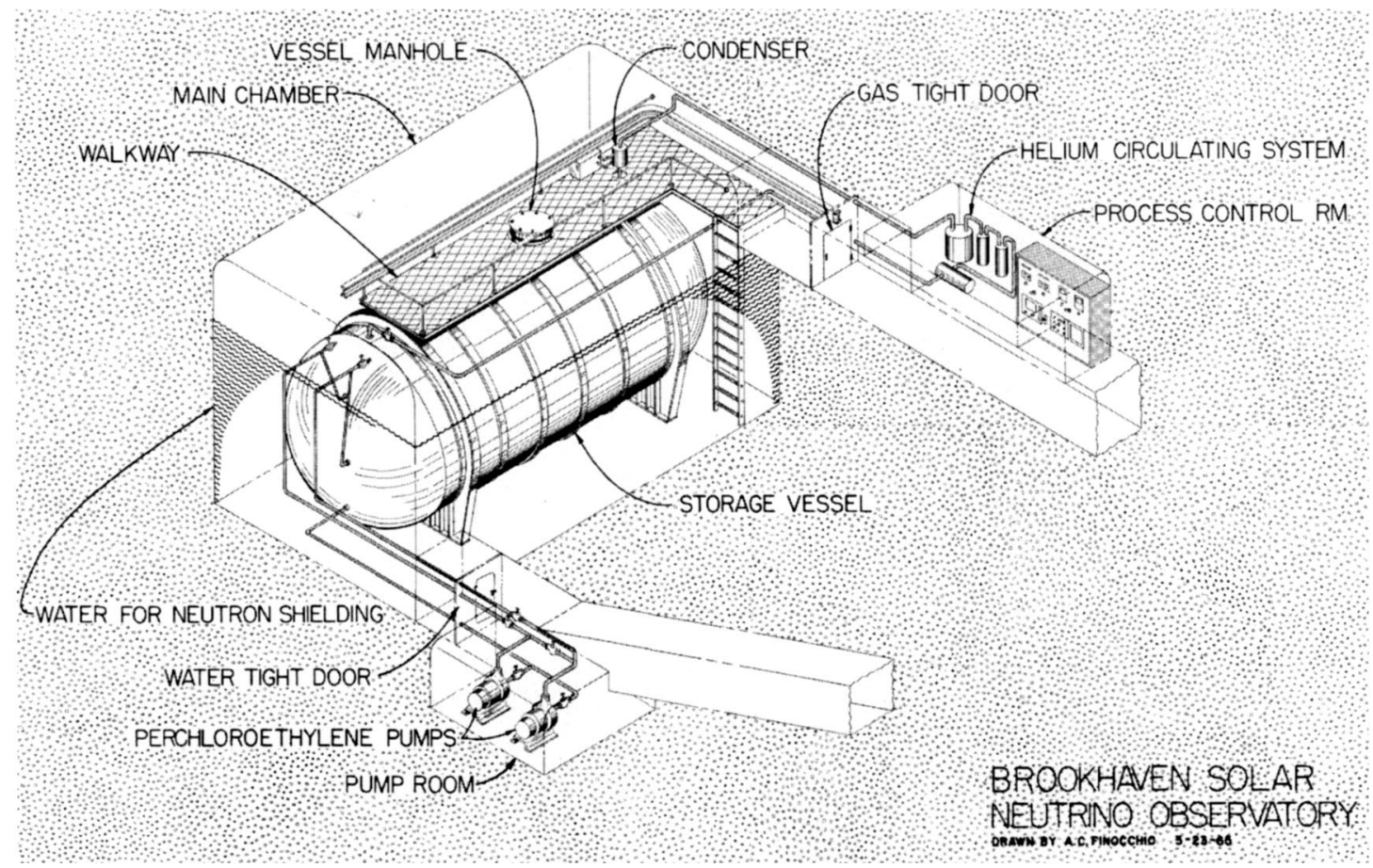

FIG. 9. A drawing of the chlorine experiment. From Sharp Bits, Spring 1969.

$\pm 0.6) \times 10^{7} \mathrm{~cm}^{-2} \mathrm{sec}^{-1}$. I hope to improve these results by improving the counter background, statistics, and longer irradiations.

Please regard these results as very preliminary. There are several points that must be checked before we are certain this is a bonafide observation. I will collect another sample in September-we are ready now, turn on the sun.

I have hopes of showing you the apparatus sometime in the future. The scenery is not be compared with the English countryside, but it has its attractions.

The measured rate was one-third that predicted by Bah-

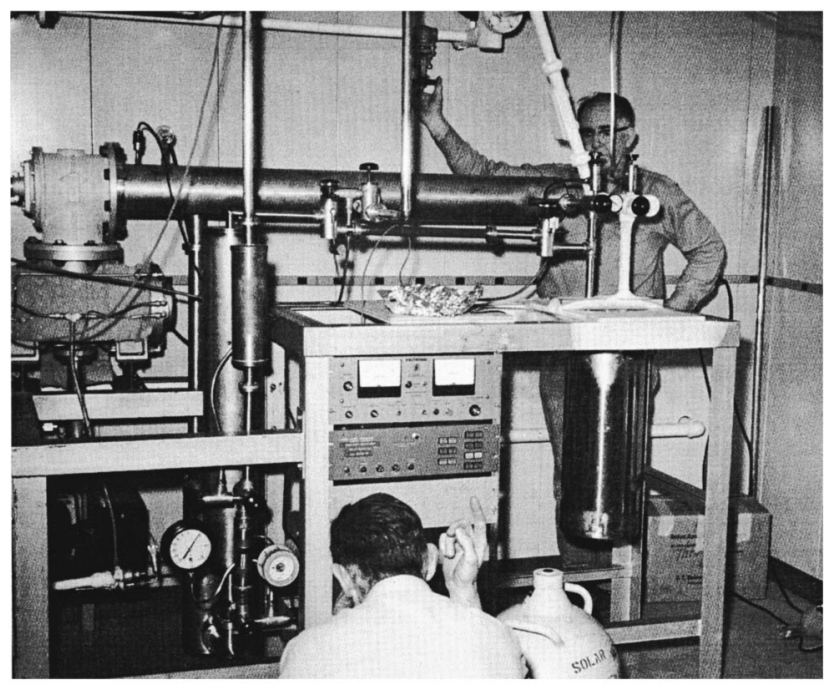

FIG. 10. Argon was trapped in a large liquid nitrogen-cooled charcoal trap under the table. From Sharp Bits, Spring 1969. call and Shaviv. You can see from my plea to Willy to "turn on the Sun" that I was already concerned.

We reported our first results in September at the American Chemical Society meeting and followed this up with a paper (Davis et al., 1968). The title of the paper was "Search for neutrinos from the sun." As everyone knows, when one uses "Search for" in the title of a paper, it means nothing was found. The upper limit was 3 SNU. In a companion paper, Bahcall et al. (1968) gave a prediction from the standard solar model of $7.5 \pm 3$ SNU. Collection of nearly 30 years of data and 30 years of refinement of the standard solar model have greatly

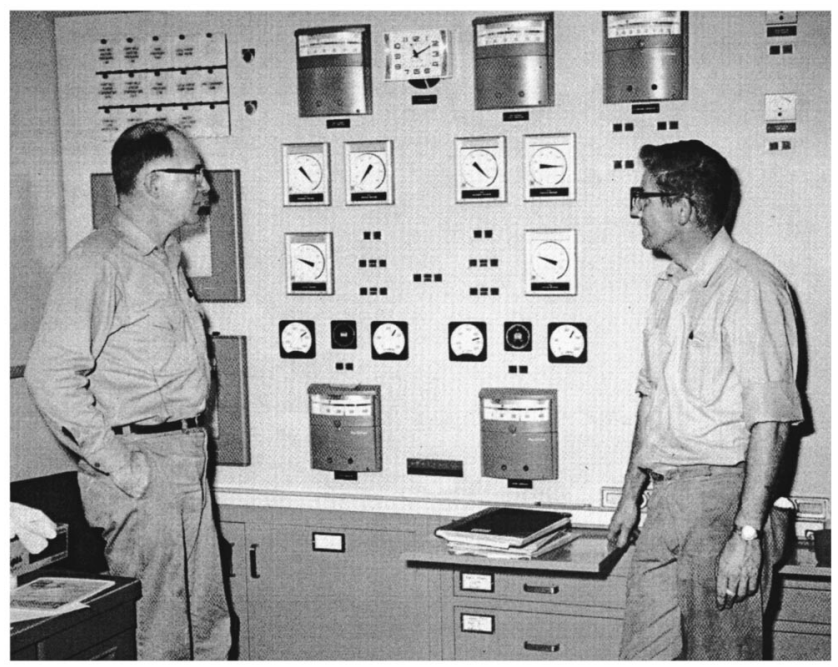

FIG. 11. John Galvin, a Brookhaven technician who worked with me for many years, and I are shown in front of the control panel. From here the entire experiment could be monitored. From Sharp Bits, Spring 1969. 


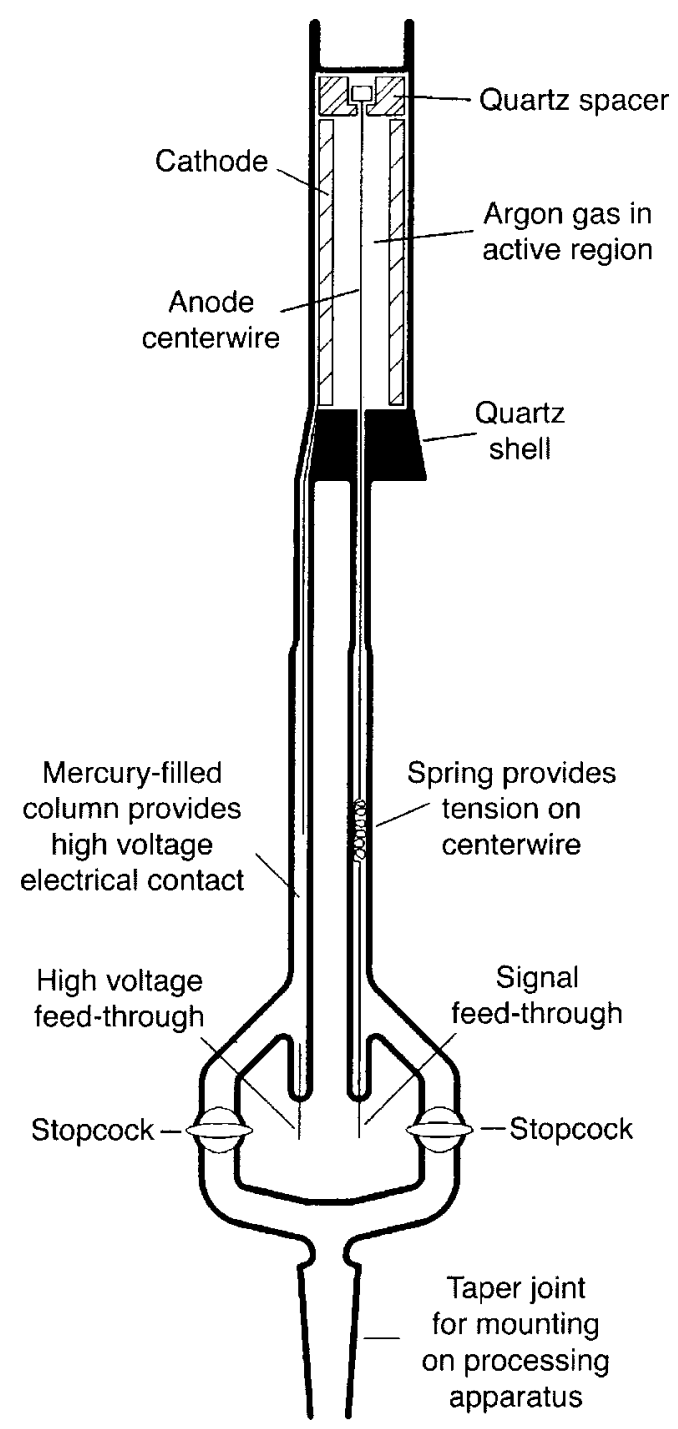

FIG. 12. Small proportional counters were used to count ${ }^{37} \mathrm{Ar}$. They typically have an overall length of $20 \mathrm{~cm}$ and an active region $30 \mathrm{~mm}$ long and $4.5 \mathrm{~mm}$ in diameter. The figure is from Cleveland et al., 1998.

improved precision: the current value from the Homestake chlorine experiment is $2.56 \mathrm{SNU}$ (Cleveland et al., 1998) and the current prediction of the standard solar model is 7.6 SNU (Bahcall et al., 2001). The numbers haven't changed much: the Sun produces one-third as many neutrinos as expected. Thus, the "solar neutrino problem" was born in 1967 and lived until the turn of the century.

Argon-37 decays by capture of a K-shell electron and emission of an Auger electron with a characteristic energy distribution. The energy spectrum from the proportional counter from Davis et al. (1968) is shown in Fig. 14. Argon from two runs in the tank and the background gave about the same number of counts in the energy region corresponding to ${ }^{37} \mathrm{Ar}$ decay. It was clear that the only way to improve the detection limit for solar neutrinos was to reduce the background.

Brookhaven electronic engineers Veljko Radeka and Lee Rogers came up with a simple, but elegant solution to this problem by devising a pulse rise-time system to

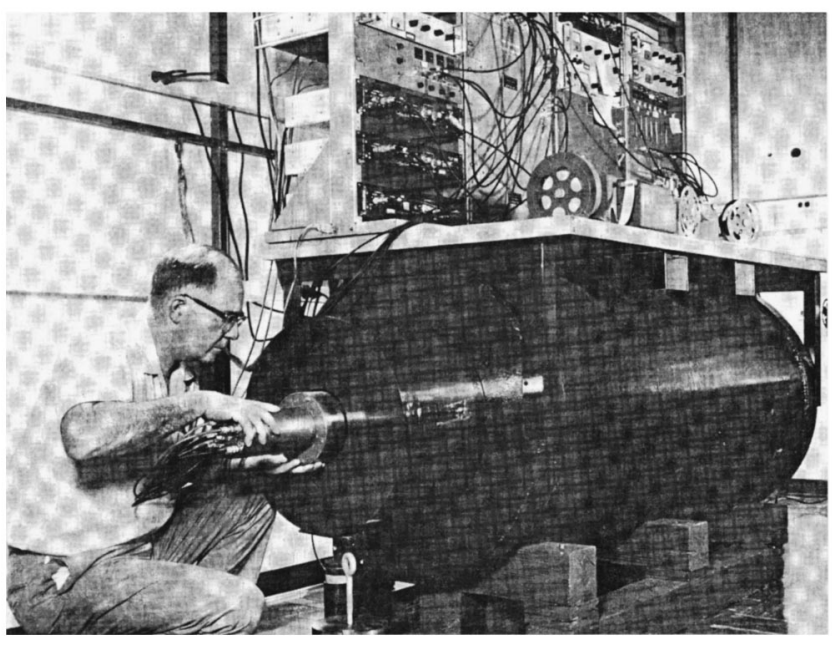

FIG. 13. In the first few years of the experiment, the counters were placed in sections of prebomb battleship gun barrels for shielding. I am shown loading a counter into the barrel. From Sharp Bits, Spring 1969.

discriminate ${ }^{37} \mathrm{Ar}$ decay events from background events. When a decay occurs in a proportional counter (Fig. 12), ion-electron pairs are made and the electrons move rapidly to the central anode, making an electrical pulse. The size of the pulse depends on the number of electrons generated, which in turn depends on the energy of the decay. The dominant decay mode of ${ }^{37} \mathrm{Ar}$, K-orbital electron capture, deposits $2.82 \mathrm{keV}$ of energy into the counting gas in the form of Auger electrons from atomic electron rearrangement. These Auger electrons have a range of about $100 \mu \mathrm{m}$, and about 100 ion electron pairs are generated. All electrons have about the same drift time, resulting in a rapidly rising pulse. The principal background is from $\gamma$-ray interactions with atoms in the counter, which generates Compton electrons. These are generated along a track passing all the way across the counter, resulting in a more slowly rising pulse. The new electronics measured not only the energy

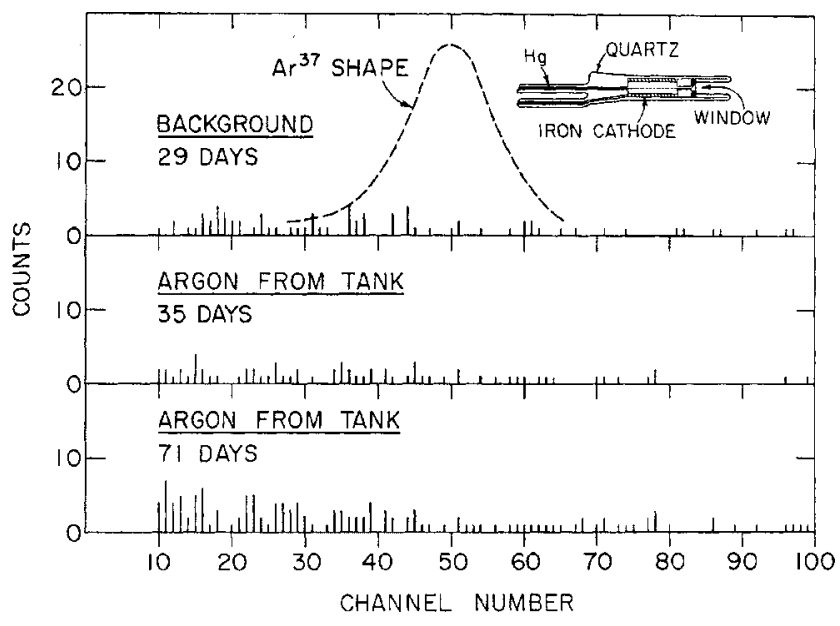

FIG. 14. Pulse-height spectra from the first two runs on the chlorine experiment. No counts in excess of background were detected in either run. Figure from Davis et al., 1968. 


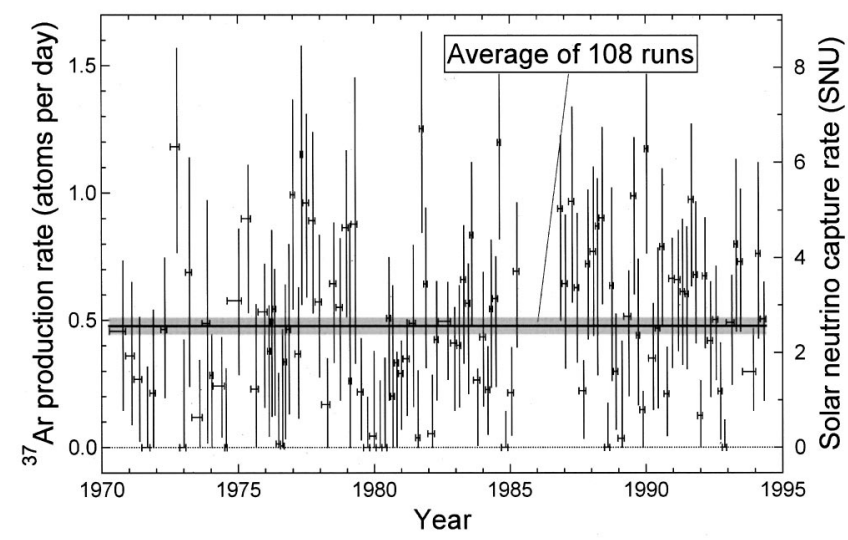

FIG. 15. A summary of all of the runs made at Homestake after implementation of rise-time counting. Background has been subtracted. Over a period of 25 years, 2200 atoms of ${ }^{37} \mathrm{Ar}$ were detected, corresponding to an average solar neutrino flux of 2.56 SNU. The gap in 1986 occurred when both perchloroethylene circulation pumps failed. Based on data from Cleveland et al., 1998.

of each decay, but also the rise time of the pulse. We began using this new system in 1970, and after about one year of observations, a clear neutrino signal was observed. We did some other things to lower backgrounds. We moved the counting apparatus to the mine, taking advantage of $\sim 1500 \mathrm{~m}$ of rock to reduce cosmic-ray backgrounds in the counters. We filled the room with water, which eliminated a minor background.

The pulse rise-time system development gave the Homestake experiment a new life. The solar neutrino production rate was indeed lower than the solar model predictions by a factor of three. The most likely explanation, in my view at the time, was that the solar model was in error. Many physicists believed that there was something wrong with our experiment. We made many tests of the experiment to improve confidence in the results and never found any experimental reason for the low neutrino flux. We introduced ${ }^{36} \mathrm{Cl}$-labeled perchloroethylene into a small tank of perchloroethylene. Chlorine-36 decays to ${ }^{36} \mathrm{Ar}$ and we recovered the expected amount of ${ }^{36} \mathrm{Ar}$. There is a reentrant tube in the big tank in Homestake. We inserted a neutron source, which makes ${ }^{37} \mathrm{Ar}$ by an $n, p$ reaction on ${ }^{37} \mathrm{Cl}$. The amount expected was recovered. We introduced 500 atoms of ${ }^{37} \mathrm{Ar}$ into the big tank and recovered them quantitatively. I was not told how much ${ }^{37} \mathrm{Ar}$ was introduced until after this check. We introduced argon as a carrier gas at the beginning of each run. We alternately used the stable isotopes ${ }^{36} \mathrm{Ar}$ or ${ }^{38} \mathrm{Ar}$, so we could check recovery in each run by mass spectrometry after ${ }^{37} \mathrm{Ar}$ counting was complete. Argon-40 is the dominant argon isotope in the atmosphere (99.6\% of total argon), so mass spectrometric measurement of the amount of ${ }^{40} \mathrm{Ar}$ tested whether there were any leaks in the apparatus. The counting efficiency of every counter was measured. These tests and more, as well as the standard operating procedure for the experiment, are described by Cleveland et al. (1998).
The solar neutrino problem lasted from 1967-2001. Over this period neither the measured flux nor the predicted flux changed significantly. I never found anything wrong with my experiment. John Bahcall never found anything wrong with the standard solar model, in fact, the advent of helioseismology confirmed the temperature profile in his model. The discrepancy between theory and experiment was a robust factor of three. Cleveland et al. (1998) summarized all of the data from the Homestake experiment. One hundred and eight runs were made after rise-time counting was implemented (Fig. 15). Over a period of 25 years, we counted a total of $2200{ }^{37} \mathrm{Ar}$ atoms and obtained a solar neutrino flux of $2.56 \pm 0.16$ (statistical error) \pm 0.16 (systematic error) SNU. The current prediction from the standard solar model (Bahcall et al., 2001) is 7.6 $6_{-1.1}^{+1.3} \mathrm{SNU}$.

The results from the Homestake experiment provoked a great deal of activity among theorists. Here are some of the more interesting and, in retrospect amusing, alternatives to the standard solar model. Fowler (1968, 1972) and Sheldon (1969) suggested that there was a secular instability in energy production in the center of the Sun. Since light takes about 10 million years to reach the surface of the Sun, while neutrinos sample the core eight minutes ago, the energy production could be low at the present time. Neutrino oscillations were suggested by Gribov and Pontecorvo (1969) and Wolfenstein (1978) and the theory was further developed by Mikheyev and Smirnov (1985) into what is now known as the MSW effect. Although neutrino oscillations now seem to be the right answer, it should be remembered that at the time most physicists viewed this as an elegant theory, but not very likely. Some of the other possibilities are more fanciful, but all from well respected physicists and astrophysicists. Libby and Thomas (1969) and Salpeter (1970) suggested that quark catalysis could play a role. Kocharov and Starbunov (1970) suggested that there was an overabundance of ${ }^{3} \mathrm{He}$ in the present Sun. Cisneros (1969) proposed that the neutrino had a significant magnetic moment. Bahcall et al. (1972) suggested that neutrinos might decay. Demarque et al. (1973) suggested that the solar interior rotated rapidly, lowering the central pressure and temperature. Prentice (1973) proposed that the Sun was in a later stage of stellar evolution, such that hydrogen was burned out and the core was made of helium. Clayton et al. (1975) proposed that the Sun's energy did not come from fusion, rather from release of energy from accretion onto a black hole at the center of the Sun.

The Homestake experiment was the only measurement of the solar neutrino flux for a long time. We had to wait 23 years for the Kamiokande experiment to confirm that the solar ${ }^{8} \mathrm{~B}$ neutrino flux was low (Hirata et al., 1990; Fukuda et al., 2001). In the 1990s, two radiochemical experiments that captured neutrinos using the inverse beta decay of ${ }^{71} \mathrm{Ga}$, SAGE (Abazov et al., 1991; Abdurashitov et al., 1999) and GALLEX (Anselmann et al., 1991; Altmann et al., 1999) showed that there was a discrepancy between the measured flux of lowerenergy neutrinos from the pp reaction and that expected 


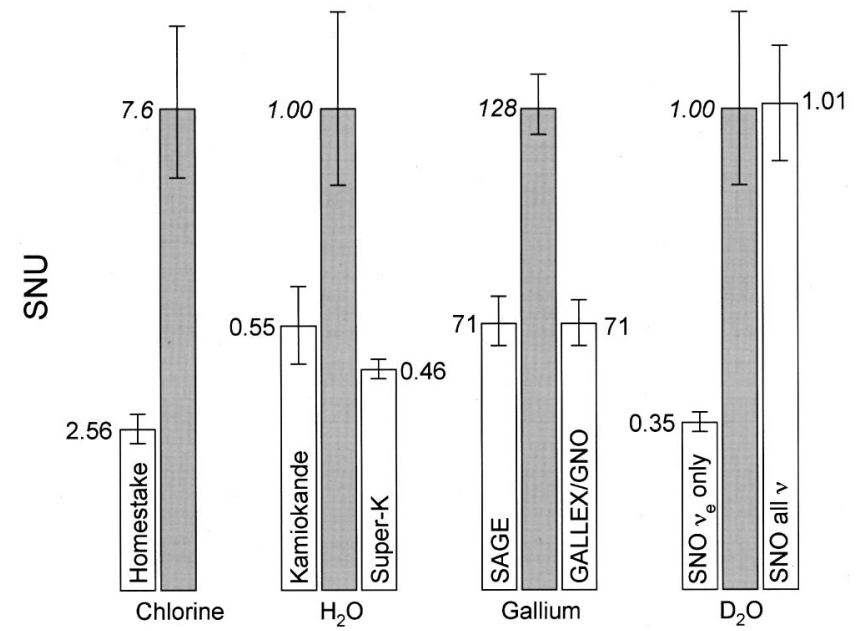

FIG. 16. A comparison of measured solar neutrino flux from the chlorine experiment, two gallium experiments (SAGE and GALLEX/GNO), two water Cerenkov experiments (Kamiokande and Super-Kamiokande) and the $\mathrm{SNO} \mathrm{D}_{2} \mathrm{O}$ experiment with theoretical predictions of Bahcall et al. (2001). The heights of the bars for theoretical predictions have been made the same to facilitate comparison with experiments. The detection of all flavors of neutrinos with the SNO experiment finally solved the "solar neutrino problem." Inspired by a similar figure of John Bahcall, http://www.sns.ias.edu/ jnb/

from the standard solar model. The gallium experiments were off by a factor of two or so.

The solar neutrino problem appears to have been solved with the first announcement of results from the Sudbury Neutrino Observatory, SNO. The MSW effect (Wolfenstein, 1978; Mikheyev and Smirnov, 1985), the possibility that neutrinos change flavor as they pass through matter, has been an attractive solution to the solar neutrino problem for several years now. The theory was given added support in 1998, when the SuperKamiokande team reported oscillations of highenergy atmospheric neutrinos from cosmic showers (Fukuda et al., 1998). In 2001, the Sudbury Neutrino Observatory team released data in which they showed that they had detected electron neutrinos and in combination with data from SuperKamiokande, showed that neutrinos oscillate between different flavors (Ahmad et al., 2001). In 2002, additional SNO data proved beyond doubt that neutrinos oscillate and that the total neutrino flux agrees with theoretical predictions (Ahmad et al., 2002).

Figure 16 shows a comparison of all solar neutrino experiments with the standard solar model. Note that only the SNO detection of all neutrinos matches the model. When we started the Homestake solar neutrino experiment, we thought we understood how the Sun worked and that a measurement of the solar neutrino flux would confirm the theory. This clearly did not turn out as planned. The collision between solar neutrino experiments and the standard solar model has ended in a spectacular way: nothing was wrong with the experiments or the theory; something was wrong with the neutrinos, in the sense that they behave in ways beyond the standard model. Neutrino astrophysics is now a vibrant field with several solar neutrino experiments now collecting data and more on the way, not to mention experiments such as KamLAND (Eguchi et al., 2003) to investigate neutrino oscillations.

\section{ACKNOWLEDGMENTS}

Brookhaven National Laboratory and later, the University of Pennsylvania, were wonderful places to carry out this research, providing not only technical support in carrying out experiments but also a stimulating intellectual environment. This work would not have been done without the support of the Atomic Energy Commission and its successor agencies and the National Science Foundation.

John Bahcall deserves special mention. He has been the keeper of the standard solar model for nearly 40 years and has been and continues to be a major force in promoting measurements of appropriate nuclear reaction rates, promoting solar neutrino experiments, and making other tests of the solar model. It is clear that without the point of reference that the standard solar model provides, there would be no solar neutrino problem. The collision of theory and experiment over the life of "the problem" made for a better experiment and better theory. I would also like to single out Kenneth Lande, who has collaborated with me for many years and, after I retired from Brookhaven in 1984, found a new home institution for me and for the Homestake experiment at the University of Pennsylvania. I would like to thank some of my other long-time collaborators: Bruce Cleveland, John Evans, John Galvin, Don Harmer, Keith Rowley, Raymond Stoenner, Jack Ullman and Paul Wildenhain. There are many others who worked with me over the years, too many to name, but all are gratefully acknowledged.

Finally, I would like to thank my son, Andrew M. Davis of the University of Chicago, for preparing and presenting this lecture.

\section{REFERENCES}

Abazov, A. I., et al., 1991, "Search for neutrinos from the Sun using the reaction ${ }^{71} \mathrm{Ga}\left(\nu_{e}, e^{-}\right){ }^{71} \mathrm{Ge}$," Phys. Rev. Lett. 67, 3332-3335.

Abdurashitov, J. N., et al., 1999, "Measurement of the solar neutrino capture rate by SAGE and implications for neutrino oscillations in vacuum," Phys. Rev. Lett. 83, 4686-4689.

Ahmad, Q. R., et al., 2001, "Measurement of the rate of $\nu_{e}$ $+d \rightarrow p+p+e^{-}$interactions produced by ${ }^{8} \mathrm{~B}$ solar neutrinos at the Sudbury Neutrino Observatory," Phys. Rev. Lett. 87, 071301.

Ahmad, Q. R., et al., 2002, "Direct evidence for neutrino flavor transformation from neutral-current interactions in the Sudbury Neutrino Observatory," Phys. Rev. Lett. 89, 011301. Altmann, M., et al., 1999, "GNO solar neutrino observations: results for GNO I,” Phys. Lett. B 490, 16-26.

Alvarez, L., 1949, "Proposed test of the neutrino theory," University of California Radiation Laboratory Report. 
Anselmann, P., et al., 1992, "Solar neutrinos observed by GALLEX at Gran Sasso,” Phys. Lett. B 285, 376-389.

Bahcall, J. N., 1962, "Electron capture and nuclear matrix elements of ${ }^{7}$ Be," Phys. Rev. 128, 1297-1301.

Bahcall, J. N., 1964, "Solar neutrinos. I. Theoretical," Phys. Rev. Lett. 12, 300-302.

Bahcall, J. N., N. Bahcall, and G. Shaviv, 1968, "Present status of the theoretical predictions for the ${ }^{37} \mathrm{Cl}$ solar-neutrino experiment," Phys. Rev. Lett. 20, 1209-1212.

Bahcall, J. N., N. Cabibbo, and A. Yahil, 1972, “Are neutrinos stable particles?" Phys. Rev. Lett. 28, 316-318.

Bahcall, J. N., M. H. Pinsonneault, and S. Basu, 2001, "Solar models: current epoch and time dependences, neutrinos, and helioseismological properties," Astrophys. J. 555, 990-1012.

Cameron, A. G. W., 1958, "Modification of the proton-proton chain,” Bull. Am. Phys. Soc. 3, 227.

Cisneros, A., 1971, "Effect of neutrino magnetic moment on solar neutrino observations," Astrophys. Space Sci. 10, 8792.

Clayton, D. D., E. Dwek, M. J. Newman, and R. J. Talbot, 1975, "Solar models of low neutrino-counting rate: the central black hole," Astrophys. J. 201, 489-493.

Cleveland, B. T., T. Daily, R. Davis, Jr., J. R. Distel, K. Lande, C. K. Lee, P. S. Wildenhain, and J. Ullman, 1998, "Measurement of the solar electron neutrino flux with the Homestake chlorine detector," Astrophys. J. 496, 505-526.

Cowan, C. L., Jr., F. Reines, F. B. Harrison, H. W. Kruse, and A. D. McGuire, 1956, "Detection of the free neutrino: a confirmation," Science 124, 103-104.

Crane, H. R., 1948, "The energy and momentum relations in the beta-decay, and the search for the neutrino," Rev. Mod. Phys. 20, 278-295.

Davis, R., Jr., 1942, "The ionization constant of carbonic acid and the solubility of carbon-dioxide in water and sodium chloride solutions from 0 to 50 degrees C," Ph.D. Dissertation (Yale University).

Davis, R., Jr., 1952, "Nuclear recoil following neutrino emission from beryllium 7," Phys. Rev. 86, 976-985.

Davis, R., Jr., 1955, “Attempt to detect the antineutrinos from a nuclear reactor by the $\mathrm{Cl}^{37}\left(\bar{\nu}, e^{-}\right) \mathrm{A}^{37}$ reaction," Phys. Rev. 97, 766-769.

Davis, R., Jr., 1958, "An attempt to observe the capture of reactor neutrinos in chlorine-37," in UNESCO Conf., Paris, Vol. 1, p. 728.

Davis, R., Jr., 1964, "Solar neutrinos. II. Experimental," Phys. Rev. Lett. 12, 303-305.

Davis, R., Jr., D. S. Harmer, and K. C. Hoffman, 1968, “A search for neutrinos from the Sun," Phys. Rev. Lett. 20, 1205-1209.

Demarque, P., J. G. Mengel, and A. V. Sweigart, 1973, "Solar rotation and the neutrino flux," Nature (London) 246, 33-35.
Eguchi, K., et al., 2003, "First results from KamLAND: evidence for reactor antineutrino disappearance," Phys. Rev. Lett. 90, 021802.

Fowler, W. A., 1958, "Completion of the proton-proton reaction chain and the possibility of energetic neutrino emission by hot stars," Astrophys. J. 127, 551-556.

Fowler, W. A., 1968, in Contemporary Physics: Trieste Symposium 1968 (International Atomic Energy Agency, Vienna), Vol. 1, p. 359.

Fowler, W. A., 1972, "What cooks with solar neutrinos?" Nature (London) 238, 24-26.

Fukuda, S., et al., 2001, "Solar ${ }^{8} \mathrm{~B}$ and hep neutrino measurements from 1258 days of Super-Kamiokande data," Phys. Rev. Lett. 86, 5651-5654.

Fukuda, Y., et al., 1998, "Evidence for oscillation of atmospheric neutrinos," Phys. Rev. Lett. 81, 1562-1567.

Gribov, V., and B. Pontecorvo, 1969, "Neutrino astronomy and lepton charge," Phys. Lett. 28B, 493-496.

Hirata, K. S., et al., 1990, "Results from one thousand days of real time, directional solar neutrino data," Phys. Rev. Lett. 65, 1297-1300.

Holmgren, H. D., and R. L. Johnston, 1958, " $\mathrm{H}^{3}(\alpha, \gamma) \mathrm{Li}^{7}$ and $\mathrm{He}^{3}(\alpha, \gamma) \mathrm{Be}^{7}$ processes," Phys. Rev. 113, 1556-1559.

Kavanagh, R. W., 1960, "Proton capture in $\mathrm{Be}^{7}$," Nucl. Phys. 15, 411-420.

Kocharov, G. E., and Yu. N. Starbunov, 1970, Proceedings of the Eleventh International Conference on Cosmic Rays, Budapest, Acta Phys. Acad. Sci. Hung. Suppl. 29,4, 353.

Libby, L. M., and F. J. Thomas, 1969, "Solar energy without neutrinos: fusion catalysis with quarks," Nature (London) 222, 1238-1240.

Mikheyev, S. P., and A. Y. Smirnov, 1985, "Resonant enhancement of oscillations in matter and solar neutrino spectroscopy," Sov. J. Nucl. Phys. 42, 913-917.

Pontecorvo, B., 1946, "Inverse $\beta^{-}$process," Chalk River Laboratory Report PD-205.

Prentice, A. J. R., 1973, "Early inhomogeneities in composition and the solar neutrino problem," Mon. Not. R. Astron. Soc. 163, 331-335.

Reines, F., 1960, "Neutrino interactions," Annu. Rev. Nucl. Sci. 10, 1-16.

Reines, F., C. L. Cowan, Jr., F. B. Harrison, A. D. McGuire, and H. W. Kruse, 1960, "Detection of the free antineutrino," Phys. Rev. 117, 159-173.

Salpeter, E. E., 1970, "Difficulties with fusion catalysis by quarks," Nature (London) 225, 165-166.

Sheldon, W. R., 1969, "Possible relation of a null solar neutrino flux to 11 year solar cycle," Nature (London) 221, 650.

Smith, P. B., and J. S. Allen, 1951, "Nuclear recoils resulting from the decay of $\mathrm{Be}^{7}$," Phys. Rev. 81, 381-385.

Wolfenstein, L., 1978, "Neutrino oscillations in matter," Phys. Rev. D 17, 2369-2374. 